\title{
Derivation of a Standardized Method to Identify the Anterior Compartment of the Lower Extremity Using Point-of-Care Ultrasonography
}

\author{
Levi T. Filler1', Daniel Orosco², Carl E. Mitchell1', John C. Porter3, Philomene Spadafore", \\ Karen J. Richey4, Bikash Bhattarai5, Marc R. Matthews4,6* (D) \\ ${ }^{1}$ Department of Emergency Medicine, Valleywise Health System, Phoenix, Arizona, USA \\ ${ }^{2}$ Department of Emergency Medicine, St. John's Regional Medical Center, Oxnard, California, USA \\ ${ }^{3}$ Department of Physical Medicine and Rehabilitation, Valleywise Health Medical Center, Phoenix, Arizona, USA \\ ${ }^{4}$ Department of Surgery, Valleywise Health Medical Center, Phoenix, Arizona, USA \\ ${ }^{5}$ Department of Medicine, Valleywise Health Medical Center, Phoenix, Arizona, USA \\ ${ }^{6}$ Arizona Burn Center, Valleywise Health Medical Center, Phoenix, Arizona, USA \\ Email: Levi_Filler@dmgaz.org, orosco.dan@gmail.com,Carl_Mitchell@dmgaz.org, John_Porter@dmgaz.org, \\ Philomene.Spadafore2@valleywisehealth.org, Karen.Richey2@valleywisehealth.org, Bikash.Bhattarai@valleywisehealth.org, \\ *marc_matthews@dmgaz.org
}

How to cite this paper: Filler, L.T., Orosco, D., Mitchell, C.E., Porter, J.C., Spadafore, P., Richey, K.J., Bhattarai, B. and Matthews, M.R. (2021) Derivation of a Standardized Method to Identify the Anterior Compartment of the Lower Extremity Using Point-of-Care Ultrasonography. Surgical Science, 12, 107-118. https://doi.org/10.4236/ss.2021.124013

Received: March 14, 2021

Accepted: April 17, 2021

Published: April 20, 2021

Copyright $\odot 2021$ by author(s) and Scientific Research Publishing Inc. This work is licensed under the Creative Commons Attribution International License (CC BY 4.0).

http://creativecommons.org/licenses/by/4.0/ (c) (i) Open Access

\begin{abstract}
Introduction: Anterior compartment syndrome (ACS) of the lower extremity is a well-recognized surgical emergency. The anterior compartment is the most frequently missed of the four compartments during lower extremity fasciotomy. This study describes a novel approach that combines sonographic measurements and physical examination landmarks to accurately identify the anterior compartment. Materials and Methods: This study was conducted as a prospective anatomical study of 94 volunteers at a single institution. Physical exam and sonographic methods were utilized to derive measurements of the anterior compartment, followed by biometric and validation data. Results: Volunteers for the derivation and validation phases of study were similar regarding gender, height and weight, age, and BMI. The derivation set revealed the distance to the anterior compartment to be $2.77 \mathrm{~cm}$ (median 2.6 $\mathrm{cm}$, range $1.5-5.4 \mathrm{~cm}$ ) from the mid-axial line and resulted in identification of the anterior compartment $100 \%$ of the time $(\mathrm{p}<0.001)$. Findings were reproducible in the internal validation set with $100 \%$ accuracy. Conclusion: The fascial planes of the anterior compartment can be identified with $100 \%$ accuracy when utilizing physical examination and sonographic methods. This can serve as a foundation for future studies evaluating ways to reduce of the number of missed anterior compartment fasciotomies in the setting of ACS.
\end{abstract}




\section{Keywords}

Acute Compartment Syndrome, Point-of-Care Ultrasound, Missed Injury, Fasciotomy

\section{Introduction}

Acute compartment syndrome (ACS) is a well-recognized condition that is universally considered a surgical emergency. ACS has an annual incidence of 7.3 per 100,000 in men and 0.7 per 100,000 in women [1] and it can result in substantial morbidity and mortality if not treated in an appropriate and timely fashion.

The exact cause of ACS has been previously debated in the literature, but it is our current understanding that extremity ACS results from the increase in the pressure within a defined muscular compartment surrounded by fascia which subsequently leads to impaired circulation, tissue ischemia and cellular anoxia [2] [3]. Tissue necrosis that develops from lack of venous and arterial flow can lead to several complications including limb deformity, ischemic muscle contracture, and amputation [1] [2]. Decompression of the affected fascial compartments via a long, cutaneous incision has been found to be the most effective and widely accepted method of treatment [2] [3] [4].

The anterior compartment of the lower extremity is the most prevalent site for ACS (62\% - 96\% [4]), and more than 70\% are due to tibia fractures [2] [4] [5] [6] [7] [8]. Additionally, roughly $2.8 \%$ of patients who sustain extremity trauma will require a fasciotomy [9] [10], and those coupled with arterial injuries will require fasciotomies even more frequently [10] [11]. Furthermore, the lower extremity anterior compartment has been found to be the most frequently missed leg compartment among surgeons when performing a fasciotomy [4]. Incomplete or missed fasciotomy can lead to considerable morbidity, including chronic lower extremity pain, neuropraxia, permanent foot drop (up to $18.2 \%$ of patients), and perfusion issues leading to amputation (12.9\% of cases) [5] [8] [10] [12] [13] [14].

The medicolegal and financial consequences of missing ACS can be substantial. One closed claim analysis review noted a mean indemnity payment of $\$ 426,000$ for cases of missed ACS, which far exceeds the average indemnity payment of $\$ 136,000$ for orthopedic surgeons malpractice claims [8]. Another review notes that about $90 \%$ of suits were related to delay in diagnosis, however, no difference was found in proportion of plantiff verdicts or in monetary awards for those cases compared with the remaining $10 \%$ of suits, which include incomplete fasciotomies [7]. This suggests that the medicolegal risks of missing a compartment during a lower extremity fasciotomy are equally as consequential, and that performing an optimal release of the lower extremity anterior compartment is essential in order to avoid these potential risks. 
The most commonly unopened compartments of the lower extremity are the anterior and the deep compartments [4]. The anterior compartment is of particular importance, as it is involved in the majority of cases of lower extremity ACS and can also be the only compartment affected in up to half of all cases [4] [15]. Additionally, the most frequent surgical revision procedures were extension of fascial incisions and decompression of unopened compartments [4] [16].

Given the above, prior emphasis has been placed primarily on tactile methods and anatomical estimations for identification of anatomic landmarks in an affected extremity which may not be completely sufficient [4]. Previous methods of identifying a precise anatomical incision site placement are varied and no standard method exists. To our knowledge, there is no currently accepted protocol or standard method related to the identification of the anterior compartment using both superficial skin landmarks combined with sonographic evaluation. Our study sought to obtain sonographic measurements using anatomic landmarks to correctly identify the optimal incision site location of the anterior compartment of the lower extremity. We anticipate that a more precise fasciotomy site incision location using point-of-care ultrasound coupled with anatomic measurements would lead to a decrease in incomplete fasciotomies during decompression. Additionally, we intended to study the effect of biometric factors in relation to our obtained compartment measurement data.

\section{Materials and Methods}

This descriptive, prospective anatomical study was conducted with 103 total volunteers at a single institution and included a derivation and validation set of data. This study protocol was reviewed and approved by the Institutional Review Board. All participants were 18 years of age and older. Our derivation set consisted of 52 volunteers, with 18 females, 25 males and a total of 86 lower extremities. We excluded 3 females and 6 males (11 lower extremities). Those excluded from the study included individuals with any prior lower extremity infection, surgery, orthopedic hardware placement, or deep venous thrombosis (Figure 1),

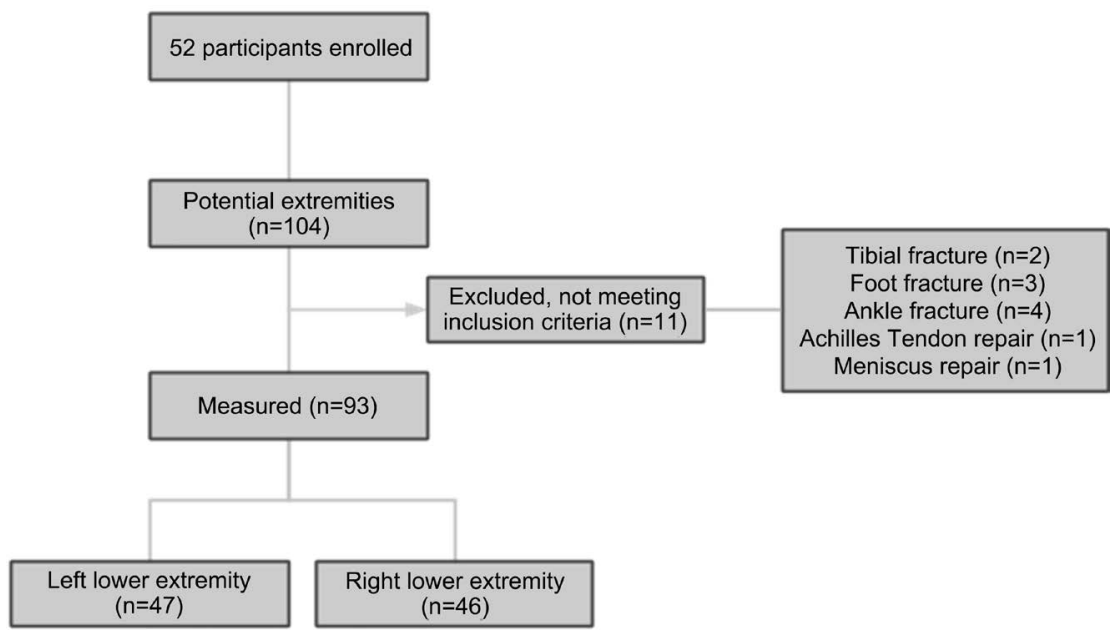

Figure 1. Derivation set inclusion and exclusion criteria. 
as these may disrupt normal anatomical fascial boundaries and tissues and cause discrepancies between extremity measurements. Our validation set consisted of a total of 51 volunteers, with 30 females, 21 males and a total of 101 lower extremities. We excluded 1 lower extremity in the validation set (lower extremity fracture). Biometric data was obtained to include age, gender, height, weight, and BMI. Leg length discrepancy and was evaluated by measuring the distance from the anterior superior iliac spine (ASIS) to the lateral and medial malleoli of each extremity. Calf circumference at the site of assessment was also obtained. A vinyl tape measure was used for all external measurements.

Measurements were taken $6 \mathrm{~cm}$ distal to the tibial tuberosity on each leg for the purpose of standardization, marking the location for sonographic assessment. First, identifiable, palpable external anatomical landmarks were obtained and marked on the skin surface, namely the axial line and the lateral edge of the tibia (ballotable surface). The axial line was denoted by drawing a line from the center of the fibular head to the center of the lateral malleolus, both identified by manual palpation. The lateral edge of the tibia was also marked (Figure 2). Once the above surface landmarks were identified by palpation and recorded, sonographic identification of the lower extremity compartments were also obtained and extrapolated to the skin surface (Figure 2).

Ultrasound measurements of the bilateral lower extremities were obtained by using the Mindray M9 ultrasound machine (Mindray North America, Mahwah, NJ) with utilization of the high frequency $(2-15 \mathrm{MHz})$ linear probe. Sonographic

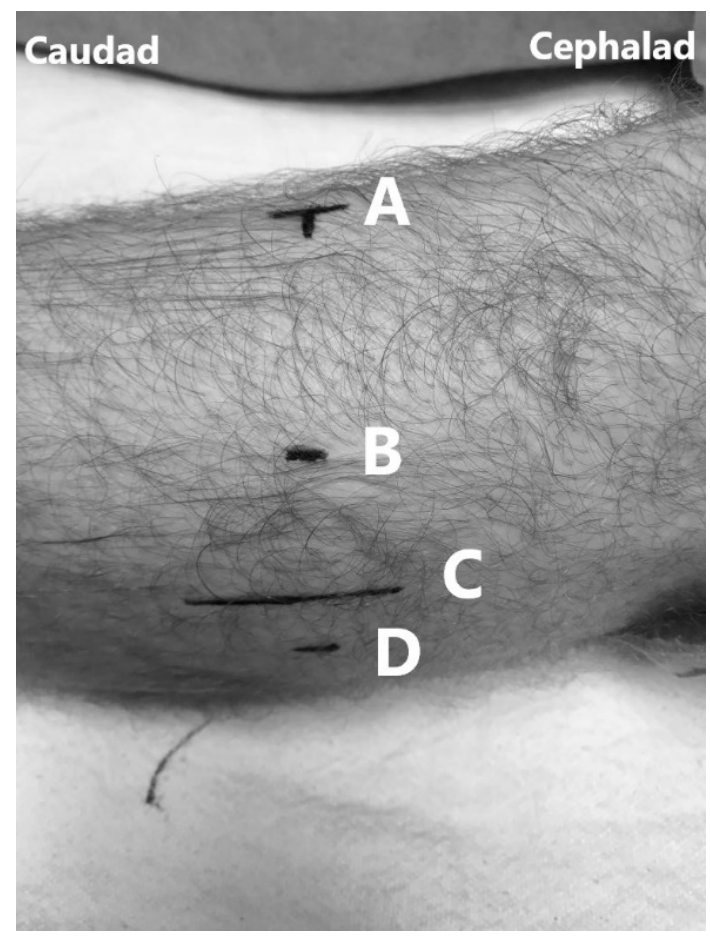

Figure 2. Left lower extremity markings obtained during derivation phase depicting the lateral edge of the tibia (A), anterior intermuscular septum (B), axial line (C), and posterior intermuscular septum (D). 
measurements were obtained by two emergency medicine trained ultrasound fellows. Ultrasound and surface measurements were validated by either an emergency medicine physician with ultrasound fellowship training, a burn surgeon, or a physiatrist. Sonographic measurements included the width of the anterior compartment (distance from the anterior inter-muscular septum to the lateral edge of the tibia bone) and the width of the lateral compartment (distance from the posterior inter-muscular septum to the anterior inter-muscular septum) (Figure 3). Once these sonographic landmarks were identified and marked on the skin surface, various distances were measured using vinyl measuring tape. These measurements included the distance from the axial line to the anterior inter-compartmental fascia, and the distance from the axial line to the lateral edge of the tibia bone, using an iterative process.

Once the initial data set was interpreted, an additional 100 lower extremities from healthy, random volunteers were measured for internal validation purposes. In the validation cohort, we used a separate group of volunteers with the same exclusion criteria. The axial line, described above, was drawn again on both limbs. From the analysis of the pilot cohort, we were able to determine that the anterior compartment would be located $2 / 3$ of the distance from the axial line to the tibial edge in all patients. With this data, we measured the distance from the axial line to the tibial edge in the validation cohort. We then calculated 2/3 of this measured distance (starting from the axial line) and evaluated this marked external location with ultrasound. We recorded the marked location and confirmed that it was indeed over the identified anterior compartment.

\section{Results}

Results are based on all 86 legs from 43 volunteers measured during the derivation phase and 101 legs from 51 volunteers during the validation phase (Table 1).

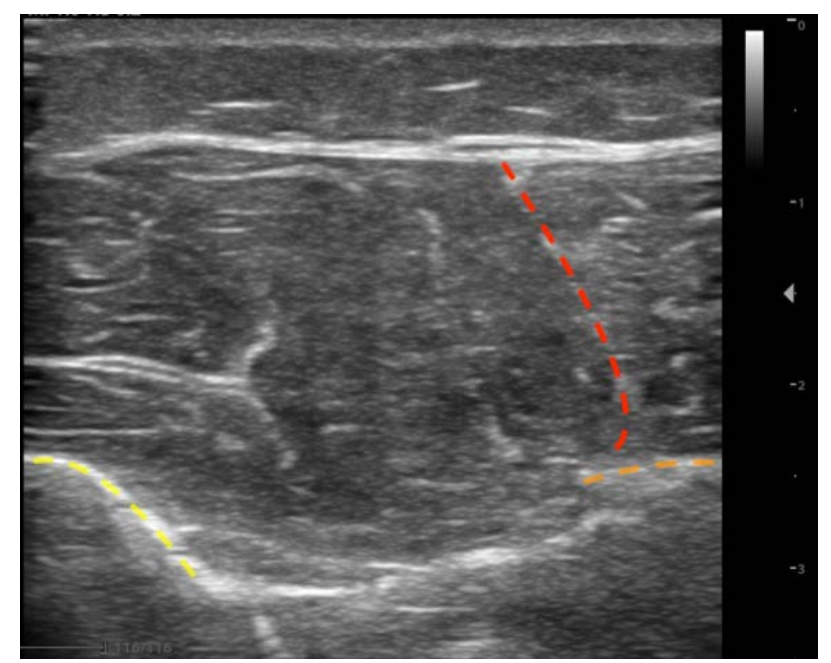

Figure 3. Transverse ultrasound image of the lower extremity anterior compartment depicting the intermuscular septum and lateral boundary of the anterior compartment (dotted red line), the lateral border of the tibia (dotted yellow line) and the interosseous membrane (dotted orange line). 
Table 1. Anatomical measurements of healthy males and female volunteers who participated in the exploratory phase of the study.

\begin{tabular}{|c|c|c|c|c|c|c|c|c|c|}
\hline & Anatomical measurements $(\mathrm{cm})$ & Sex & Mean & SD & Median & Min & $\operatorname{Max}$ & $\mathrm{P}^{*}$ & $\mathrm{P}^{\star *}$ \\
\hline \multirow{4}{*}{\multicolumn{2}{|c|}{$\begin{array}{l}\text { Anterior compartment width } \\
\text { (lateral edge of the tibia to fascia) }\end{array}$}} & All & 4.8 & 0.7 & 4.5 & 3.5 & 7.0 & 0.030 & 0.003 \\
\hline & & Female & 4.5 & 0.6 & 4.5 & 3.5 & 5.5 & & \\
\hline & & Male & 5.0 & 0.8 & 4.7 & 3.5 & 7.0 & & \\
\hline & & All & 5.3 & 0.9 & 5.0 & 3.0 & 7.3 & 0.262 & 0.164 \\
\hline \multirow{3}{*}{\multicolumn{2}{|c|}{ Lateral compartment width }} & Female & 5.1 & 1.0 & 5.0 & 3.0 & 7.3 & & \\
\hline & & Male & 5.4 & 0.8 & 5.5 & 4.0 & 7.0 & & \\
\hline & & All & 2.9 & 0.6 & 2.8 & 1.8 & 5.4 & 0.058 & 0.010 \\
\hline \multirow{3}{*}{\multicolumn{2}{|c|}{ Axial line to the anterior compartment }} & Female & 3.1 & 0.9 & 2.9 & 1.8 & 5.4 & & \\
\hline & & Male & 2.7 & 0.4 & 2.7 & 1.8 & 3.5 & & \\
\hline & & All & 7.4 & 0.9 & 7.4 & 6.0 & 10 & 0.216 & 0.979 \\
\hline \multirow[t]{17}{*}{ Right leg } & Axial line to the lateral edge of the tibia & Female & 7.2 & 1.0 & 7.0 & 6.0 & 10.0 & & \\
\hline & & Male & 7.6 & 0.8 & 7.5 & 6.5 & 10.0 & & \\
\hline & & All & 37.8 & 3.4 & 38.0 & 30.7 & 46.5 & $<0.005$ & 0.307 \\
\hline & Calf circumference $6 \mathrm{~cm}$ distal to tibial tuberosity & Female & 35.6 & 3.4 & 35.5 & 30.7 & 46.5 & & \\
\hline & & Male & 39.4 & 2.4 & 39.0 & 35.0 & 45.5 & & \\
\hline & & All & 89.2 & 6.8 & 90.5 & 72.0 & 100.5 & $<0.005$ & 0.236 \\
\hline & Anterior superior iliac spine to medial malleolus & Female & 83.7 & 5.3 & 84.0 & 72.0 & 95.0 & & \\
\hline & & Male & 93.2 & 4.7 & 94.0 & 80.7 & 100.5 & & \\
\hline & Anterior superior iliac spine to lateral malleolus & Female & 85.0 & 5.3 & 84.0 & 73.5 & 96.0 & & \\
\hline & & Male & 95.6 & 4.4 & 96.0 & 83.0 & 102.0 & & \\
\hline & & All & 5.0 & 0.7 & 5.0 & 4.0 & 6.5 & 0.010 & \\
\hline & $\begin{array}{l}\text { Anterior compartment width } \\
\text { (lateral edge of the tibia to fascia) }\end{array}$ & Female & 4.6 & 0.5 & 4.5 & 4.0 & 6.0 & & \\
\hline & & Male & 5.2 & 0.7 & 5.0 & 4.0 & 6.5 & & \\
\hline & & All & 5.2 & 0.8 & 5.0 & 3.2 & 7.5 & 0.756 & \\
\hline & Lateral compartment width & Female & 5.1 & 0.9 & 5.0 & 3.2 & 7.0 & & \\
\hline & & Male & 5.2 & 0.8 & 5.0 & 4.0 & 7.5 & & \\
\hline & & All & 2.6 & 0.5 & 2.5 & 1.5 & 3.9 & 0.078 & \\
\hline \multirow[t]{8}{*}{ Left leg } & Axial line to the anterior compartment & Female & 2.8 & 0.6 & 2.7 & 2.0 & 3.9 & & \\
\hline & & Male & 2.5 & 0.5 & 2.5 & 1.5 & 3.9 & & \\
\hline & & All & 7.4 & 0.9 & 7.5 & 5.3 & 10 & 0.155 & \\
\hline & Axial line to the lateral edge of the tibia & Female & 7.2 & 1.0 & 7.0 & 5.3 & 9.5 & & \\
\hline & & Male & 7.6 & 0.9 & 7.6 & 5.5 & 10.0 & & \\
\hline & & All & 38.0 & 3.5 & 38.0 & 30.7 & 49 & 0.005 & \\
\hline & Calf circumference $6 \mathrm{~cm}$ distal to tibial tuberosity & Female & 36.1 & 4.0 & 35.0 & 30.7 & 49.0 & & \\
\hline & & Male & 39.3 & 2.3 & 39.0 & 35.0 & 45.0 & & \\
\hline
\end{tabular}




\begin{tabular}{cccccccc}
\hline & All & 89.4 & 6.9 & 90.0 & 69.5 & 100 & $<0.005$ \\
Anterior superior iliac spine to medial malleolus & Female & 84.0 & 5.9 & 83.8 & 69.5 & 96.5 & \\
& Male & 93.3 & 4.5 & 93.0 & 80.8 & 100.0 & \\
& All & 91.3 & 7.1 & 92.0 & 70.5 & 102 & $<0.005$ \\
Anterior superior iliac spine to lateral malleolus & Female & 85.4 & 6.1 & 84.3 & 70.5 & 98.0 & \\
& Male & 95.5 & 4.1 & 95.5 & 85.0 & 102.0 & \\
\hline
\end{tabular}

*Students $\mathrm{t}$-test P-less than 0.005 indicates the measurements are different between males and females. ${ }^{*}$ Paired $\mathrm{t}$-test comparing left and right leg measurements.

The average (median, range) distance from axial line to the anterior intercompartmental fascia was $2.7 \mathrm{~cm}(2.6,1.5-5.4)$. Based on the iterative evaluation of multiple possible distances from the axial line to lateral edge of tibia, an proposed incision made at $1 / 2$ this distance would be within the anterior compartment $98 \%$ of the time, whereas an incision at $2 / 3$ this distance would result in $100 \%$ of incisions being within the anterior compartment. Given that the mean distance from the axial line to the tibial crest was $7.4 \mathrm{~cm}$, this would place the proposed incision point at a mean distance of $2.5 \mathrm{~cm}$ lateral to the tibial crest, however, given that the distance from the axial line to the tibial crest will vary among individuals and extremities, the $2 / 3$ distance metric was utilized. Our validation set with the point of interest at $2 / 3$ the distance from the axial line to the lateral edge of the tibia confirmed these findings. Measurements noted did not differ substantially between the left and right legs of the same individual, except for the anterior compartment $(\mathrm{P}=0.003)$ and the axial line to the anterior compartment distance $(P=0.010)$. Although statistically significant, the magnitude of differences in mean measurements for these two distances was $0.3 \mathrm{~cm}$ or less between the left and right legs. This subtle difference was deemed negligible to potentially cause measurement error when determining the incision site in actual patients.

Our results were consistent regardless of demographics, including age, BMI, gender, and extremity laterality (Table 2). Volunteers for both the exploration and validation phases of study were generally similar in terms of gender $(\mathrm{P}=$ 0.101), distribution of underweight, normal, overweight or obese volunteers $(\mathrm{P}=$ $0.399)$ as well as in terms of age $(\mathrm{P}=0.086)$ and BMI (0.182). Additionally, volunteer attributes in both the exploratory and validation phases were similar, which further strengthens generalizability.

\section{Discussions}

Accurately identifying and accessing the lower extremity anterior compartment is crucial to avoid unnecessary patient harm and medicolegal risks. Given the nature of ACS, identification of the anterior compartment and fasciotomy incision site has previously relied heavily on palpable external skin landmarks, with a multitude of various locations being previously used in clinical practice. 
Table 2. Age and general physical measurements of the healthy volunteers who participated in two phases of the study.

\begin{tabular}{|c|c|c|c|c|c|}
\hline \multirow[b]{2}{*}{ Phase } & & \multicolumn{3}{|c|}{ Mean (median, range) of the measurements } & \multirow[t]{2}{*}{ P-value ${ }^{\star}$} \\
\hline & & Female & Male & All & \\
\hline \multirow{4}{*}{ Exploration $(n=43)^{* *}$} & Age & $35.8(31,22-63)$ & $34.1(31,24-62)$ & $34.8(31,22-63)$ & 0.099 \\
\hline & Weight & $70.8(63,47.6-158)$ & $95.6(93.4,64-194)$ & $85.2(83.5,47.6-194)$ & 0.943 \\
\hline & Height & $153.8(161.4,1.6-177.8)$ & $166(180.3,1.7-193)$ & $160.8(175.2,1.6-193)$ & 0.707 \\
\hline & BMI & $24.4(23.5,19.4-33.9)$ & $28.3(27.9,22.1-40.2)$ & $26.7(25.5,19.4-40.2)$ & 0.272 \\
\hline \multirow{4}{*}{ Validation $(\mathrm{n}=51)^{\star \star}$} & Age & $39.4(38.5,27-65)$ & $37.7(35,22-65)$ & $38.7(36,22-65)$ & \\
\hline & Weight & $76(73.9,49.4-136.5)$ & $92.7(89.4,58.5-122.1)$ & $82.9(84.4,49.4-136.5)$ & \\
\hline & Height & $164.9(162.6,147.3-180.3)$ & $179.4(180.3,167.4-193)$ & $170.9(172.2,147.3-193)$ & \\
\hline & BMI & $27.9(26.6,18.1-50.1)$ & $28.7(28.3,20.9-37.4)$ & $28.2(27.8,18.1-50.1)$ & \\
\hline
\end{tabular}

${ }^{\star}$ Comparing differences between exploration and validation phases based on Wilcoxon two-sample test with $\mathrm{t}$-approximation.

Furthermore, surgical training varies amongst general, burn, trauma, plastic and orthopedic training programs with educational inconsistency amongst surgical technique. Our study attempted to identify an optimal and practical method for evaluating the proper incision site over the anterior compartment using ultrasonography as an adjunct to palpable skin landmarks, of which can easily be identified at the bedside, while also avoiding distances that would prove harmful to vascular and neurologic structures, such as deep arterial perforators and the common peroneal nerve. We found that an incision that is placed $2 / 3$ the distance from the axial line (contrived line from the center of the fibular head to the center of the lateral malleolus) to the lateral edge of the tibia, yields the most optimal location to access the anterior compartment.

This proposed distance is of utmost importance given the currently accepted standard practice of evaluating the lower extremity in preparation for an anterior compartment fasciotomy. Currently, a four-compartment fasciotomy is recommended in the lower leg to treat ACS [17]. Various two incision methods have been previously described, although, no official standard exists. There are two generally accepted techniques that have been described regarding the decompression of the anterior and peroneal compartments [16] [18]. One technique describes placing a skin incision directly over the inter-muscular septum, between the anterior and peroneal compartments with a separate deep incision releasing the fascia directly over each compartment [16] [18] [19]. An alternative approach places the skin incision much more anteriorly to decompress the anterior compartment directly by incising the fascia exactly in line with the skin incision, about $1.5-2 \mathrm{~cm}$ lateral to the tibial crest [16] [18] [20] [21]. The muscle bulk is then retracted to allow decompression of the lateral compartment by a separate incision through the intermuscular septum. Although the latter method is effective, it risks placing an incision too close to the tibial crest, which can increase the risk of common peroneal nerve injury and disrupt arterial perforator supply to the area [18]. 
The widely accepted distance of $2 \mathrm{~cm}$ lateral to the tibial crest has been adopted in order to avoid potential injury to anterior and peroneal perforators, which may be jeopardized with a more anterior or posterior incision, respectively; however, literature regarding this distance is limited and ambiguous [18]. According to Pallister et al. (2016), anterior perforators were found to lie lateral to the incision line, a median distance of $2.2 \mathrm{~mm}$ (range $2-2.9 \mathrm{~cm}$ ) from the tibial crest. The median distance of the peroneal perforators from this incision line was $6.2 \mathrm{~cm}$ (range $4.2-9 \mathrm{~cm}$ ) [18]. Our study places a proposed incision site at $2.5 \mathrm{~cm}$ (mean and median distance) lateral to the tibial crest, well within the anterior compartment, correlating approximately with the previously proposed safe distance of $2 \mathrm{~cm}$ lateral to the tibial crest as well as being outside of the median distance of the anterior arterial perforators assessed by Pallister et al. (2016).

Furthermore, the mean distance from the tibial crest to the anterior compartmental fascia (anterior compartment width) in our study was $4.8 \mathrm{~cm}$ and 5.0 $\mathrm{cm}$ in the right and left legs, respectively. This distance of the anterior compartment boundary may allow for an additional 2.3 and $2.5 \mathrm{~cm}$ of safe incision site distance from the tibial crest to the anterior compartmental fascia on the right and left leg, respectively, thus avoiding the anterior arterial perforators of the anterior compartment.

The use of point-of-care ultrasound (POCUS) to identify the anatomy of the lower extremity was paramount to our study and was used to validate our findings. The use of ultrasound to delineate the structures of the musculoskeletal system is well-documented throughout the literature [22]. Point-of-care ultrasound has been shown throughout the literature to be a useful tool when diagnosing various musculoskeletal pathology, identifying structures and aiding various musculoskeletal procedures [23].

\section{Limitations}

During study development and implementation, we identified limitations to our investigation. Our research focused exclusively on the anterior compartment of the lower extremity; thus, we cannot extrapolate our findings to other extremities or compartments of the leg. Additionally, vascular arterial perforators and the common peroneal nerve were not directly identified during our observations, both of which may be injured during anterior compartment fasciotomy [24]. Vigilant surgical care is still required with a fasciotomy incision to avoid vascular and nervous injuries. Furthermore, our study population included healthy adult volunteers, which may not correlate with findings found in those adult or pediatric patients with ACS who might have sustained traumatic injuries leading to an anatomical distortion. One cadaver study did show that certain fascial angles do change with increased compartmental pressure via saline infusion, however, whether this changes fascial distances to any substantial or clinically significant degree has yet to be seen [24]. Further studies are recommended going forward in the trauma and pediatric populations. 
Our study describes a novel technique of determining the optimal location to perform an anterior compartment fasciotomy that would lead to successful identification $100 \%$ of the time. Further studies are warranted to further identify the optimal location for fasciotomy of the anterior compartment in the setting of ACS while also mitigating risks to important vascular and neurologic structures. Our findings can serve as a foundation for additional studies in the future that evaluate ways to reduce the number of missed anterior compartment fasciotomies in the setting of ACS.

\section{Acknowledgements}

We gratefully acknowledge Vernon L. Yeager, PhD. (retired, professor of anatomy) and Jay M. Crutchfield (general surgeon) for their suggestions and guidance in the preparation of this study and the manuscript.

\section{Conflicts of Interest}

The study authors have disclosed that they do not have any conflicts of interest.

\section{References}

[1] Volkmann, R. (1881) Ischämische Muskellähmungen und -kontrakturen [Ischemic Muscle Paralysis and Contractures]. Zentralbl Chir., 8, 801-803.

[2] Wind, T.C., Saunders, S.M., Barfield, W.R., Mooney, J.F. and Hartsock, L.A. (2012) Compartment Syndrome after Low-Energy Tibia Fractures Sustained during Athletic Competition. Journal of Orthopaedic Trauma, 26, 33-36. https://doi.org/10.1097/BOT.0b013e3182163367

[3] McQuillan, W.M. and Nolan, B. (1968) Ischaemia Complicating Injury. A Report of Thirty-Seven Cases. The Journal of Bone and Joint Surgery (British), 50-B, 482-492. https://doi.org/10.1302/0301-620X.50B3.482

[4] Ritenour, A.E., Dorlac, W.C., Fang, R., et al. (2008) Complications after Fasciotomy Revision and Delayed Compartment Release in Combat Patients. The Journal of Trauma: Injury, Infection, and Critical Care, 64, S153-S162. https://doi.org/10.1097/TA.0b013e3181607750

[5] Tzioupis, C., Cox, G. and Giannoudis, P.V. (2009) Acute Compartment Syndrome of the Lower Extremity: An Update. Orthopaedics and Trauma, 23, 433-440. https://doi.org/10.1016/j.mporth.2009.09.003

[6] Mcqueen, M.M., Gaston, P. and Court-Brown, C.M. (2000) Acute Compartment Syndrome WHO IS AT RISK? The Journal of Bone and Joint Surgery (British), 82-B, No. 2. https://doi.org/10.1302/0301-620X.82B2.0820200

[7] DePasse, J.M., Sargent, R., Fantry, A.J., Bokshan, S.L., Palumbo, M.A. and Daniels, A.H. (2017) Assessment of Malpractice Claims Associated with Acute Compartment Syndrome. Journal of the American Academy of Orthopaedic Surgeons, 25, e109-e113. https://doi.org/10.5435/JAAOS-D-16-00460

[8] Bhattacharyya, T. and Vrahas, M.S. (2004) The Medical-Legal Aspects of Compartment Syndrome. The Journal of Bone \& Joint Surgery, 86, 864-868. https://doi.org/10.2106/00004623-200404000-00029

[9] Branco, B.C., Inaba, K., Barmparas, G., et al. (2011) Incidence and Predictors for the Need for Fasciotomy after Extremity Trauma: A 10-Year Review in a Mature Level I 
Trauma Centre. Injury, 42, 1157-1163. https://doi.org/10.1016/j.injury.2010.07.243

[10] Cone, J. and Inaba, K. (2017) Lower Extremity Compartment Syndrome. Trauma Surgery \& Acute Care Open, 2, e000094. https://doi.org/10.1136/tsaco-2017-000094

[11] Farber, A., Tan, T.-W., Hamburg, N.M., et al. (2012) Early Fasciotomy in Patients with Extremity Vascular Injury Is Associated with Decreased Risk of Adverse Limb Outcomes: A Review of the National Trauma Data Bank. Injury, 43, 1486-1491. https://doi.org/10.1016/j.injury.2011.06.006

[12] Badhe, S., Baiju, D., Elliot, R., Rowles, J. and Calthorpe, D. (2009) The "Silent" Compartment Syndrome. Injury, 40, 220-222.

https://doi.org/10.1016/j.injury.2008.07.023

[13] Heemskerk, J. and Kitslaar, P. (2003) Acute Compartment Syndrome of the Lower Leg: Retrospective Study on Prevalence, Technique, and Outcome of Fasciotomies. World Journal of Surgery, 27, 744-747.

[14] Lollo, L. and Grabinsky, A. (2016) Clinical and Functional Outcomes of Acute Lower Extremity Compartment Syndrome at a Major Trauma Hospital. International Journal of Critical Illness \& Injury Science, 6, 133-142.

https://doi.org/10.4103/2229-5151.190648

[15] Hope, M.J. and McQueen, M.M. (2004) Acute Compartment Syndrome in the Absence of Fracture. Journal of Orthopaedic Trauma, 18, 220-224. https://doi.org/10.1097/00005131-200404000-00005

[16] Kashuk, J.L., Moore, E.E., Pinski, S., et al. (2009) Lower Extremity Compartment Syndrome in the Acute Care Surgery Paradigm: Safety Lessons Learned. Patient Safety in Surgery, 3, Article No. 11. https://doi.org/10.1186/1754-9493-3-11

[17] Köstler, W., Strohm, P.C. and Südkamp, N.P. (2004) Acute Compartment Syndrome of the Limb. Injury, 35, 1221-1227.

https://doi.org/10.1016/j.injury.2004.04.009

[18] Pallister, I., Morris, R.M., Lloyd, T., et al. (2016) A Novel Method to Correctly Place the Fasciotomy Incision for Decompression of the Anterior and Peroneal Compartments of the Leg. Injury, 47, 962-968.

https://doi.org/10.1016/j.injury.2016.02.007

[19] Rorabeck, C.H. (1984) The Treatment of Compartment Syndromes of the Leg. The Journal of Bone and Joint Surgery—Series B, 66-B, No. 1. https://doi.org/10.1302/0301-620X.66B1.6693486

[20] Court-Brown, C.M., Cross, A.T., Hahn, D.M., et al. (1997) A Report by the British Orthopaedic Association/British Association of Plastic Surgeons Working Party on the Management of Open Tibial Fractures September 1997. British Journal of Plastic Surgery, 50, 570-583. https://doi.org/10.1016/S0007-1226(97)90501-4

[21] Clodius, L. (2010) J. Nanchahal, S. Nayagam, U. Kahn, Ch. Moran, S. Barrett, F. Sanderson, I Pallister, Standards for the Management of Open Fractures of the Lower Limb. European Journal of Plastic Surgery, 33, 321. https://doi.org/10.1007/s00238-010-0484-7

[22] Balius, R., Bong, D.A., Ardèvol, J., Pedret, C., Codina, D. and Dalmau, A. (2016) Ultrasound-Guided Fasciotomy for Anterior Chronic Exertional Compartment Syndrome of the Leg. Journal of Ultrasound in Medicine, 35, 823-829. https://doi.org/10.7863/ultra.15.04058

[23] Situ-LaCasse, E., Grieger, R.W., Crabbe, S., Waterbrook, A.L., Friedman, L. and Adhikari, S. (2018) Utility of Point-of-Care Musculoskeletal Ultrasound in the Evaluation of Emergency Department Musculoskeletal Pathology. World Journal of 
Emergency Medicine, 9, 262-266.

https://doi.org/10.5847/wjem.j.1920-8642.2018.04.004

[24] Mühlbacher, J., Pauzenberger, R., Asenbaum, U., et al. (2019) Feasibility of Ultrasound Measurement in a Human Model of Acute Compartment Syndrome. World Journal of Emergency Surgery, 14, Article No. 4.

https://doi.org/10.1186/s13017-019-0222-9 